\title{
Building an evidence base for the primary care workforce
}

\author{
Vari M Drennan professor of healthcare and policy research ${ }^{1}$, Simon de Lusignan professor of \\ primary care and clinical informatics ${ }^{2}$, Heather Gage professor of health economics ${ }^{2}$, Jon Gabe \\ professor of sociology ${ }^{3}$, Mary Halter associate professor ${ }^{1}$
}

1Joint Faculty of Kingston University and St George's University of London, London SW17 ORE, UK; ${ }^{2}$ University of Surrey, Guildford GU2 7XH, UK;
${ }^{3}$ Royal Holloway University of London, Egham TW20 OEX, UK

Building an evidence base for the effects of different types of staff in general practice is challenging and takes time.

McCartney is right to say that no evidence shows that physician associates make a difference to clinician stress and burnout. ${ }^{1}$ But more evidence is available than she suggests, which may be important at a time of considerable vacancies for general practitioners and practice nurses.

We found that physician associates were safely attending a younger, less complex group of patients than GPs. ${ }^{2}$ These were patients requesting same day or urgent appointments with problems classified mainly as minor and self limiting. Surveyed patients reported high levels of satisfaction with their consultations with both physician associates and GPs.

Interviewed patients reported willingness to consult a physician associate, contingent on their assessment of the severity of the problem and desire for provider continuity. ${ }^{3}$

McCartney expressed concern that physician associates might interrupt a GP for prescription signature. We found that this was done in ways to avoid interrupting.

Finally, our interviews with GPs (some employing physician associates and some not) found two viewpoints on how best to manage clinical workload and to deploy staff in general practice. ${ }^{4}$ One view was that "doctor first"-whereby the GP attends all patients and then delegates activities to members of the team-was the most efficient. Others thought that GPs should attend the more complex patients and those with medically acute problems, while other team members, with supervisory support, attended those with more minor, self limiting problems.

Our research indicates that physician associates can contribute safely to skill mixed general practice teams. As such they have the potential to be an asset to the primary care workforce in the face of doctor shortages, increasing demand, and financial stringency.

Competing interests: None declared.

Full response at: http://www.bmj.com/content/359/bmj.j5022/rr-3.

McCartneyM. Margaret McCartney: Are physician associates just "doctors on the cheap"?BMJ2017;359:j5022. doi:10.1136/bmj.j502229158238

2 DrennanVMHalterMBrearleyS. Investigating the contribution of physician assistants to primary care in England: a mixed-methods study. Health Services and Delivery Research2014;2. doi:10.3310/hsdr0216025642506

3 HalterMDrennanVMJolyLMGabeJGageHde LusignanS. Patients' experiences of consultations with physician associates in primary care in England: A qualitative study Health Expect2017;20:1011-9. https://doi.org/10.1111/hex.12542.

doi:10.1111/hex.1254228429886

4 DrennanVMGabeJHalterMde LusignanSLevensonR. Physician associates in primary health care in England: A challenge to professional boundaries?Soc Sci Med2017;181:9-16. doi:10.1016/j.socscimed.2017.03.04528364578

Published by the BMJ Publishing Group Limited. For permission to use (where not already granted under a licence) please go to http://group.bmj.com/group/rights-licensing/ permissions 